ESAIM: PROCEEDINGS, March 2012, Vol. 35, p. 174-183

Fédération Denis Poisson (Orléans-Tours) et E. Trélat (UPMC), Editors

\title{
PROBABILISTIC INTERPRETATION FOR THE NONLINEAR POISSON-BOLTZMANN EQUATION IN MOLECULAR DYNAMICS.
}

\author{
NICOLAS PERRIN ${ }^{1}$
}

\begin{abstract}
The Poisson-Boltzmann (PB) equation describes the electrostatic potential of a biomolecular system composed by a molecule in a solvent. The electrostatic potential is involved in biomolecular models which are used in molecular simulation. In consequence, finding an efficient method to simulate the numerical solution of $\mathrm{PB}$ equation is very useful. As a first step, we establish in this paper a probabilistic interpretation of the nonlinear PB equation with Backward Stochastic Differential Equations (BSDEs). This interpretation requires an adaptation of existing results on BSDEs.
\end{abstract}

Résumé. En dynamique moléculaire, l'équation de Poisson-Boltzmann (PB) permet de décrire le potentiel électrostatique d'un système moléculaire composé d'une molécule dans un solvant. Ce potentiel électrostatique intervient dans les modèles de simulation numérique permettant de comprendre la structure, la dynamique et le fonctionnement des protéines. La résolution numérique de l'équation de PB est donc une étape importante de ces simulations. Aussi, nous proposons dans un premier temps, une interprétation probabiliste de l'équation de PB non-linéaire à l'aide des Equations Différentielles Stochastiques Rétrogrades (EDSR). Cette interprétation nécessite une adaptation des résultats d'existence et d'unicité des solutions d'EDSR.

\section{INTRODUCTION}

In this paper, we establish a probabilistic interpretation for the solution of the nonlinear Poisson-Boltzmann (PB) equation

$$
-\nabla \cdot(\epsilon(x) \nabla u(x))+\kappa^{2}(x) \sinh \{u(x)\}=g(x), \quad x \in \mathbb{R}^{3},
$$

where $\epsilon$ and $\kappa$ are piecewise constant functions from $\mathbb{R}^{3}$ to $(0,+\infty)$, and $g$ is a singular measure. Our probabilistic interpretation consists in the sum of a singular known function and the solution of a particular Backward Stochastic Differential Equation (BSDE). We prove an existence and uniqueness result for this BSDE by extending to our situation the methodology developed in $\left[\mathrm{BDH}^{+} 03\right]$ or [Par99].

The PB equation is used to calculate electrostatic energies and forces in molecular simulation. The numerical methods which are already used are Finite Element, Boundary Element or Finite Difference ( $c f$. [BBC06] for a discussion on these methods). The probabilistic interpretation we state in this work should provide theoretical foundations for the use of BSDEs simulation methods.

In Section 1, we introduce the Poisson-Boltzmann equation in molecular dynamics and underline three difficulties we have to face to get a probabilistic interpretation of the nonlinear Poisson-Boltzmann equation.

In Section 2, we recall the probabilistic interpretation of divergence-form operators with discontinuous coefficients in term of SDE with weighted local time.

\footnotetext{
1 TOSCA project-team, INRIA Sophia Antipolis, France. nicolas.perrin@inria.fr
} 
In Section 3, we very briefly introduce BSDEs theory, before giving a general result for the existence and uniqueness of the solution of BSDEs with time-dependent monotonicity (Theorem 3.3), which we apply in order to get a probabilistic interpretation theorem (Theorem 3.5) of the solution of the corresponding Partial Differential Equation (PDE).

In Section 4, we state our main result (Theorem 4.1). In order to prove it, we state an existence, uniqueness and regularity theorem (Theorem 4.2) for the solution $v$ in $H^{1}\left(\mathbb{R}^{d}\right)$ to the regularised Poisson-Boltzmann equation. Then we conclude in Section 4.3 by giving the steps of proof for our main result.

\section{The Poisson-Boltzmann equation in Molecular Dynamics}

The electrostatic potential of a biomolecular system in a medium of electric permittivity $\epsilon$ generated by a charge distribution $\rho_{c}$ is given by the Poisson equation (cf. [BBC06])

$$
-\nabla \cdot(\epsilon(x) \nabla u(x))=4 \pi \rho_{c}(x), \quad x \in \mathbb{R}^{3} .
$$

We consider a molecule immersed in an aqueous solvent. The charge distribution $\rho_{c}$ can be decomposed as a sum of the molecule contribution $\rho_{m}$ and the solvent contribution $\rho_{s}$. The contribution of the molecule is modelled by a finite system of charges centred in the positions of the $N$ atoms of the molecule

$$
\rho_{m}(x)=\sum_{i=1}^{N} q_{i} \delta_{x_{i}}(x), \quad x \in \mathbb{R}^{3},
$$

where $q_{i} \in \mathbb{R}$ and $x_{i} \in \mathbb{R}^{3}$ are respectively the charge and the position of the $i$ th atom and where $\delta_{x}$ is the Dirac distribution at $x \in \mathbb{R}^{3}$. The solvent contribution is modelled by:

$$
\rho_{s}(x)=e_{c} \sum_{j=1}^{m} \bar{n}_{j} z_{j} \exp \left\{-e_{c} z_{j} u(x) / k_{B} T_{e}-V_{j}(x) / k_{B} T_{e}\right\}, \quad x \in \mathbb{R}^{3},
$$

where $m$ is the number of different types of ions in the solvent, $\bar{n}_{j}$ is the number of ions of the type $j, z_{j}$ is the valence of ions of the type $j, e_{c}$ is the elementary charge, $k_{B}$ is the Boltzmann constant, $T_{e}$ is the system temperature and $V_{j}$ models the steric interaction between the ions of the type $j$ and the molecule.

For an electrolyte as $\mathrm{NaCl}$ where $N=2$ with $q_{1}=1$ and $q_{2}=-1$, the Poisson equation simplifies to give the nonlinear Poisson-Boltzmann equation

$$
-\nabla \cdot(\epsilon(x) \nabla u(x))+8 \pi e_{c} \bar{n} e^{-V(x) / k_{B} T_{e}} \sinh \left\{e_{c} u(x) / k_{B} T_{e}\right\}=4 \pi \sum_{i=1}^{N} q_{i} \delta_{x_{i}}(x), \quad x \in \mathbb{R}^{3} .
$$

Let $\Omega_{\text {int }}$ and $\Omega_{\text {ext }}$ be two disjoint open subsets of $\mathbb{R}^{3}$, which model the molecule and the solvent respectively, such that $\bar{\Omega}_{\text {int }} \cup \bar{\Omega}_{\text {ext }}=\mathbb{R}^{3} ; \Omega_{\text {int }}$ is supposed to be bounded with a smooth boundary $\Gamma$.

The dielectric permittivity $\epsilon$ is represented by a discontinuous function taking two values inside the molecule $\Omega_{\text {int }}$ (vacuum permittivity) and outside $\Omega_{\text {ext }}$ (solvant permittivity):

$$
\epsilon(x)= \begin{cases}\epsilon_{\mathrm{int}}>0 & \text { si } x \in \Omega_{\mathrm{int}} \\ \epsilon_{\mathrm{ext}}>0 & \text { si } x \in \Omega_{\mathrm{ext}}\end{cases}
$$

In the following, we study the nonlinear Poisson-Boltzmann equation

$$
-\nabla \cdot(\epsilon(x) \nabla u(x))+\kappa^{2}(x) \sinh \{u(x)\}=g(x), \quad x \in \mathbb{R}^{3} .
$$


where the modified Debye-Hückel parameter

$$
\kappa^{2}(x)= \begin{cases}0 & \text { si } x \in \Omega_{\text {int }} \\ \bar{\kappa}^{2}>0 & \text { si } x \in \Omega_{\text {ext }}\end{cases}
$$

approximates $e^{-V(\cdot) / k_{B} T_{e}} \frac{8 \pi e_{c}^{2} I}{k_{B} T_{e}}$ with $I=\frac{1}{2} \sum_{s} \bar{n}_{s} z_{s}^{2}$. We should emphasize that this approximation requires a hypothesis of low linear charge density as underlined in [BBC06].

The probabilistic interpretation for the nonlinear PB equation we propose in this paper requires to handle three difficulties:

(1) singularity of the source term (cf. Section 2.1);

(2) discontinuity of the coefficient in the divergence-form operator (cf. Section 2.2);

(3) non-linearity of the equation (cf. Section 3).

Remark 1.1. If we suppose $e_{c} u(\cdot)$ much smaller than $k_{B} T_{e}$, we obtain a linear version of the Poisson-Boltzmann equation

$$
-\nabla \cdot(\epsilon(x) \nabla u(x))+\kappa^{2}(x) u(x)=g(x), \quad x \in \mathbb{R}^{3},
$$

for which a probabilistic interpretation has been established in [BCMT10].

\section{HANDLING OF THE SINGULARITY OF THE SOURCE TERM AND THE DIVERGENCE-FORM OPERATOR}

In this section, we handle the singularity of the source term and discontinuity of the coefficient in the divergence-form operator.

\subsection{The Poisson-Boltzmann equation with a regularised source terme}

The singular source term is handled by writing the solution as a sum of a known singular function and the solution of a regularised equation.

The following regularisation is introduced in [BCMT10]. Let $\chi$ be a $\mathcal{C}^{\infty}$ function with a compact support embedded in $\Omega_{\text {int }}$, such that $\chi(x)=1$ for $x$ close to $\left\{x_{1}, \ldots, x_{N}\right\}$ and $G=\sum_{i} G_{i}$ where

$$
G_{i}(x)=\frac{q_{i}}{\epsilon_{\text {int }}} \frac{1}{\left|x-x_{i}\right|}, \quad x \in \mathbb{R}^{3},
$$

satisfying

$$
-\nabla \cdot\left(\epsilon_{\mathrm{int}} \nabla G_{i}\right)=q_{i} \delta_{i}
$$

We then study the function $v=u-\chi G$ which is the solution of

$$
-\nabla \cdot(\epsilon(x) \nabla v(x))+\kappa^{2}(x) \sinh (v(x))=\tilde{g}(x), \quad x \in \mathbb{R}^{3} .
$$

The regularised source term is then expressed as $\tilde{g}(x)=\epsilon_{\mathrm{int}}(G(x) \Delta \chi(x)+\nabla G(x) . \nabla \chi(x))$ for $x \in \mathbb{R}^{3}$, since the support of $\chi G$ is in $\Omega_{\text {int }}$ while $\kappa$ is null on $\Omega_{\text {int }}$. In particular, the function $\tilde{g}$ is $\mathcal{C}^{\infty}$.

\subsection{The martingale problem associated with the divergence-form operator}

In [BCMT10], a probabilistic interpretation is introduced for divergence-form operators in $\mathbb{R}^{d}, d \geqslant 1$ with discontinuous coefficients of the form

$$
\mathcal{L} \cdot=\nabla(\epsilon(x) \nabla \cdot)
$$

where $\epsilon$ is a piecewise constant function from $\mathbb{R}^{d}$ to $(0, \infty)$ with a smooth discontinuity manifold $\Gamma$.

We suppose that $\Omega_{\text {int }}$ is a simply connected set with a boundary $\Gamma$ of class $\mathcal{C}^{\infty}$, and that $\Omega_{\text {ext }}=\mathbb{R}^{d} \backslash\left(\Omega_{\text {int }} \cup \Gamma\right)$. The smoothness of $\Gamma$ allows us to construct a mapping $\pi$ of class $\mathcal{C}_{b}^{2}$ (where $\mathcal{C}_{b}^{2}$ is the set of 2 time continuously 


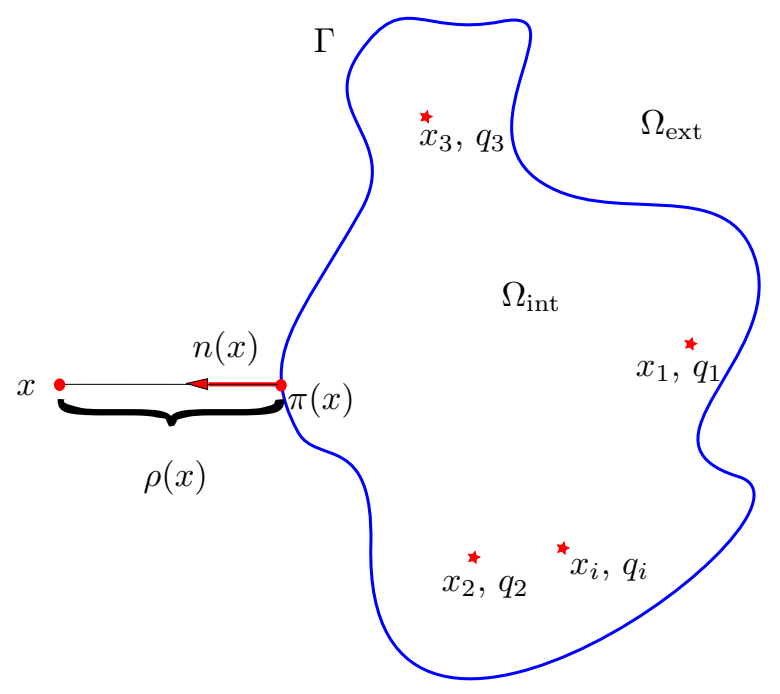

Figure 1

differentiable functions with bounded derivatives of all order up to 2) from a neighborhood $\mathcal{N}$ of $\Gamma$ to $\Gamma$ such that

$$
|x-\pi(x)|=d(x, \Gamma), \quad \forall x \in \mathcal{N},
$$

where $d(x, \Gamma)$ denotes the distance of $x$ to $\Gamma$. Let $n(x)$ be the unit vector normal to $\Gamma$ at $x \in \Gamma$ pointing in direction of $\Omega_{\text {ext }}$. Finally, let $\rho$ be the signed distance to $\Gamma$ positive in $\Omega_{\text {ext }}$ and negative in $\Omega_{\text {int }}$ for all $x \in \mathcal{N}$

$$
\rho(x)=(x-\pi(x)) \cdot n(\pi(x)), \quad x \in \mathcal{N},
$$

which can be extended to the whole Euclidean space $\mathbb{R}^{d}$ in a function $C_{b}^{2}$ still denoted $\rho$ (cf. Fig. 1).

The probabilistic interpretation of elliptic and parabolic PDEs driven by the operator $\mathcal{L}$ relies on the following martingale problem.

Definition 2.1. Let $(\mathcal{C}, \mathcal{B})$ be the set $\mathcal{C}$ of continuous functions $w$ from $[0, \infty)$ to $\mathbb{R}^{d}$ endowed with the Borel cylindrical $\sigma$-field $\mathcal{B}$ and the canonical filtration $\left(\mathcal{B}_{t}, t \geqslant 0\right)$. A family of probability measures $\mathbb{P}_{x}$ on $(\mathcal{C}, \mathcal{B})$ solves the martingale problem for the operator $\mathcal{L}$ if, for all $x \in \mathbb{R}^{d}$, one has

$$
\mathbb{P}_{x}\{w \in \mathcal{C}: w(0)=x\}=1,
$$


and, for all $\phi$ satisfying

$$
\begin{gathered}
\phi \in \mathcal{C}_{b}^{0}\left(\mathbb{R}^{d}\right) \cap \mathcal{C}_{b}^{2}\left(\mathbb{R}^{d} \backslash \Gamma\right), \\
\epsilon \nabla \phi \cdot(n \pi) \in \mathcal{C}_{b}^{0}(\mathcal{N})
\end{gathered}
$$

the process

is a $\mathbb{P}_{x^{-}}\left(\mathcal{B}_{t}\right)$ martingale.

$$
M_{t}^{\phi}(w):=\phi(w(t))-\phi(w(0))-\int_{0}^{t} \mathcal{L} \phi(w(s)) \mathrm{d} s
$$

It is shown in [BCMT10] that the unique solution of this martingale problem is the law $\mathbb{P}_{x}$ of all weak solution $\left(\Omega, \mathcal{F}^{x},\left(\mathcal{F}_{t}^{x}\right)_{t \geqslant 0}, \mathbb{P}_{x},\left\{B_{t} ; t \geqslant 0\right\},\left\{X_{t}^{x} ; t \geqslant 0\right\}\right)$ of the Stochastic Differential Equation (SDE)

$$
\left\{\begin{array}{l}
X_{t}^{x}=x+\int_{0}^{t} \sqrt{2 \epsilon\left(X_{s}^{x}\right)} \mathrm{d} B_{s}+\frac{\epsilon_{\mathrm{ext}}-\epsilon_{\mathrm{int}}}{2 \epsilon_{\mathrm{ext}}} \int_{0}^{t} n\left(X_{s}^{x}\right) \mathrm{d} L_{s}^{0}\left(D^{x}\right) \\
D_{t}^{x}=\rho\left(X_{t}^{x}\right)
\end{array}\right.
$$

where $B$ is a $\mathcal{F}_{t}^{x}$-standard $d$-dimensional Brownian motion, and $L_{s}^{0}\left(D^{x}\right)$ is the local time at 0 of the continuous semimartingale $D^{x}$. It is actually shown in [BCMT10] that all weak solutions to the SDE (6) are identical in law. The above process $X^{x}$ is used in the new probabilistic interpretation that we develop in next section.

\section{A Probabilistic interpretation in terms of BSDES}

One of the probabilistic methods to handle nonlinear Partial Differential Equations (PDEs) is based on Backward Stochastic Differential Equations (BSDEs) (cf. [BDH ${ }^{+} 03$ ] or [Par99]). We give a result for BSDEs with a time-dependent monotonicity condition. Then we conclude by applying the above result to BSDEs driven by a SDE with weighted local time.

\subsection{BSDEs with time-dependent monotonicity condition}

In all what follows, we consider $B$ be a standard $d$-dimensional Brownian motion defined on a probability space $(\Omega, \mathcal{F}, \mathbb{P})$, where $\{\mathcal{F}\}$ is the natural augmented filtration of $B$. Let $\tau$ be a $\mathcal{F}_{t}$ stopping time not necessarily inite, $\xi$ a $\mathcal{F}_{\tau}$-measurable random variable and $f$ an application from $\Omega \times \mathbb{R}^{+} \times \mathbb{R}^{k} \times \mathbb{R}^{k \times d}$ to $\mathbb{R}^{k}$.

Definition 3.1. A solution of the $\operatorname{BSDE}(\tau, \xi, f)$ is a couple of progressively measurable processes $(Y, Z)$ with values in $\mathbb{R}^{k} \times \mathbb{R}^{d \times k}$, which satisfies the following properties

(1) $t \rightarrow \mathbf{1}_{\{t \leqslant \tau\}} f\left(t, Y_{t}, Z_{t}\right)$ is in $L_{\mathrm{loc}}^{1}(0, \infty)$, and $t \rightarrow Z_{t}$ is in $L_{\mathrm{loc}}^{2}(0, \infty)$;

(2) $Y_{t \wedge \tau}=Y_{T \wedge \tau}+\int_{t \wedge \tau}^{T \wedge \tau} f\left(s, Y_{s}, Z_{s}\right) \mathrm{d} s-\int_{t \wedge \tau}^{T \wedge \tau} Z_{s} \mathrm{~d} B_{s}$ for all $0 \leqslant t \leqslant T$;

(3) $Y_{t}=\xi$ and $Z_{t}=0$ on the event $\{t \geqslant \tau\}$.

The application $f$ is called the generator of the BSDE.

Let suppose that:

(h1) (a) $f(\cdot, y, z)$ is progressively measurable for all $y$ and $z$;

(b) for all $r>0$, for all $n \in \mathbb{N}^{\star}, \sup _{|y| \leqslant r}|f(t, y, 0)-f(t, 0,0)| \in L^{1}(] 0, n[\times \Omega, \mathrm{d} t \otimes \mathbb{P})$;

(c) Lipschitz continuity : $\left|f(t, y, z)-f\left(t, y, z^{\prime}\right)\right| \leqslant K(t)\left\|z-z^{\prime}\right\|$ for all $t, y, z, z^{\prime}$ a.s., where $K$ is a progressively measurable positive process which is upper bounded by a constant $\bar{K}$,

(d) monotonicity : for all $t, y, y^{\prime}, z$ a.s.,

$$
\left\langle y-y^{\prime}, f(t, y, z)-f\left(t, y^{\prime}, z\right)\right\rangle \leqslant \mu(t)\left|y-y^{\prime}\right|^{2}
$$

where $\mu$ is a progressively measurable process which is upper bounded by a constant $\bar{\mu}$ and lower bounded by a constant $\underline{\mu}$, 
(e) integrability :

$$
\mathbb{E}\left[\int_{0}^{\tau} e^{\int_{0}^{t} \lambda(s) \mathrm{d} s}|f(t, 0,0)|^{2} \mathrm{~d} t\right]<\infty
$$

where $\lambda$ is a progressively measurable process such that, for all $t$ in $\mathbb{R}^{+}$,

$$
2 \mu(t)+K^{2}(t)<\lambda(t)
$$

(h2) the final condition $\xi$ is a $\mathcal{F}_{\tau}$-measurable random variable such that

$$
\mathbb{E}\left[\int_{0}^{T} e^{\int_{0}^{t} \lambda(s) \mathrm{d} s}\left|f\left(t, e^{-1 / 2 \int_{0}^{t} \tilde{\lambda}(s) \mathrm{d} s} \bar{\xi}_{t}, e^{-1 / 2 \int_{0}^{t} \tilde{\lambda}(s) \mathrm{d} s} \bar{\eta}_{t}\right)\right|^{2} \mathrm{~d} t\right]<\infty
$$

where $\tilde{\lambda}(t):=2 \mu(t)+K^{2}(t), \bar{\xi}=e^{\int_{0}^{T} \tilde{\lambda}(s) \mathrm{d} s} \xi, \bar{\xi}_{t}=\mathbb{E}\left[\bar{\xi} \mid \mathcal{F}_{t}\right]$ and $\bar{\eta}$ are predictable processes such that

$$
\begin{gathered}
\bar{\xi}=\mathbb{E}[\bar{\xi}]+\int_{0}^{\infty} \bar{\eta}_{t} \mathrm{~d} B_{t}, \\
\mathbb{E}\left[\int_{0}^{\infty}\left|\bar{\eta}_{t}\right|^{2} \mathrm{~d} t\right]<\infty .
\end{gathered}
$$

Definition 3.2. For a given progressively measurable process $\lambda$, the solution $(Y, Z)$ of $\operatorname{BSDE}(\tau, \xi, f)$ is said to be in $L_{\lambda(\cdot)}^{2}$ if

$$
\|(Y, Z)\|_{\lambda(\cdot)}^{2}:=\mathbb{E}\left[\sup _{0 \leqslant t \leqslant \tau} e^{\int_{0}^{t} \lambda(s) \mathrm{d} s}\left|Y_{t}\right|^{2}+\int_{0}^{\tau} e^{\int_{0}^{t} \lambda(s) \mathrm{d} s}\left(\left|Y_{t}\right|^{2}+\left\|Z_{t}\right\|^{2}\right) \mathrm{d} t\right]<\infty .
$$

We prove in [Per12] the following existence and uniqueness result of the solution to BSDEs.

Theorem 3.3. If Assumptions (h1-h2) are true, there exists a unique solution $(Y, Z)$ to the BSDE $(\tau, \xi, f)$ such that, for all progressively measurable process $\lambda$ such that $\lambda(\cdot)-2 \mu(\cdot)-K^{2}(\cdot)>\bar{\lambda}>0$,

$$
\|(Y, Z)\|_{\lambda(\cdot)}^{2} \leqslant C \mathbb{E}\left[e^{\int_{0}^{\tau} \lambda(s) \mathrm{d} s}|\xi|^{2}+\int_{0}^{\tau} e^{\int_{0}^{t} \lambda(s) \mathrm{d} s}|f(t, 0,0)|^{2} \mathrm{~d} t\right] .
$$

The proof of this theorem follows closely the steps of the proof in [Par99] by adapting them to time dependent coefficients.

\subsection{BSDEs driven by SDEs with weighted local time}

Let $\left(\Omega, \mathcal{F}^{x},\left(\mathcal{F}_{t}^{x}\right)_{t \geqslant 0}, \mathbb{P}_{x},\left\{B_{t} ; t \geqslant 0\right\},\left\{X_{t}^{x} ; t \geqslant 0\right\}\right)$ be an arbitrary weak solution of the SDE (6). Consider the Forward-Backward $\operatorname{SDE}(\infty, 0, f)$, for all $t, T$, such that for all $0 \leqslant t \leqslant T$,

$$
Y_{t}^{x}=Y_{T}^{x}+\int_{t}^{T} f\left(X_{s}^{x}, Y_{s}^{x}, Z_{s}^{x}\right) \mathrm{d} s-\int_{t}^{T} Z_{s}^{x} \mathrm{~d} B_{s}
$$

where $f$ is a generator from $\mathbb{R}^{d} \times \mathbb{R}^{k} \times \mathbb{R}^{k \times d}$ to $\mathbb{R}^{k}$ such that

(H1) $f(\cdot, y, z)$ is measurable for all $y$ and $z$;

(H2) $y \rightarrow f(x, y, z)$ is continuous for all $x, z$;

(H3) Lipschitz continuity : $\left|f(x, y, z)-f\left(x, y, z^{\prime}\right)\right| \leqslant K(x)\left\|z-z^{\prime}\right\|$ for all $x, y, z, z^{\prime}$ a.s., where $K$ is measurable,positive and upper bounded by a constant $\bar{K}$, 
(H4) monotonicity : for all $x, y, y^{\prime}, z$ a.s.,

$$
\left\langle y-y^{\prime}, f(x, y, z)-f\left(x, y^{\prime}, z\right)\right\rangle \leqslant \mu(x)\left|y-y^{\prime}\right|^{2}
$$

where $\mu$ is measurable, upper bounded by a constant $\bar{\mu}$ and lower bounded by a constant $\underline{\mu}$,

(H5) integrability :

$$
\mathbb{E} \int_{0}^{\infty} e^{\int_{0}^{t} \lambda\left(X_{s}^{x}\right) \mathrm{d} s}\left|f\left(X_{t}, 0,0\right)\right|^{2} \mathrm{~d} t<\infty
$$

where $\lambda$ is measurable and such that $2 \mu(x)+K^{2}(x)<\lambda(x)$ for all $x$ in $\mathbb{R}^{d}$,

(H6) $\sup _{|y| \leqslant r}\left|f\left(X_{t}^{x}, y, 0\right)-f\left(X_{t}^{x}, 0,0\right)\right| \in L^{1}(] 0, n[\times \Omega, \mathrm{d} t \otimes \mathbb{P})$, for all $n \in \mathbb{N}^{*}$ and $r>0$.

The following existence and uniqueness theorem for Eq. (8) is a corollary of Theorem 3.3 with $\tau=\infty$ and $\xi=0$.

Theorem 3.4. If Assumptions (H1-H6) are true, the BSDE (8) has a unique solution (in the sense of Definition 3.1) such that

$$
\begin{aligned}
& \mathbb{E}\left[\sup _{0 \leqslant t} e^{\int_{0}^{t} \lambda\left(X_{s}^{x}\right) \mathrm{d} s}\left|Y_{t}^{x}\right|^{2}+\int_{0}^{\infty} e^{\int_{0}^{t} \lambda\left(X_{s}^{x}\right) \mathrm{d} s}\left(\left|Y_{t}^{x}\right|^{2}+\left\|Z_{t}^{x}\right\|^{2}\right) \mathrm{d} t\right] \\
\leqslant & C \mathbb{E}\left[\int_{0}^{\infty} e^{\int_{0}^{t} \lambda\left(X_{s}^{x}\right) \mathrm{d} s}\left|f\left(X_{t}^{x}, 0,0\right)\right|^{2} \mathrm{~d} t\right] .
\end{aligned}
$$

Sketch of proof. The key arguments of the proof are the following:

- The weak uniqueness of the solution $\left(\Omega, \mathcal{F}^{x},\left(\mathcal{F}_{t}^{x}\right)_{t \geqslant 0}, \mathbb{P}_{x},\left\{B_{t} ; t \geqslant 0\right\},\left\{X_{t}^{x} ; t \geqslant 0\right\}\right)$ to the SDE (6) allows one to apply Theorem 4.29 in [JS03] to $\left(\Omega, \mathcal{F}^{x},\left(\mathcal{F}_{t}^{x}\right)_{t \geqslant 0}, \mathbb{P}_{x}\right)$ local martingales to get a process $\left\{\tilde{Z}_{t}^{x} ; t \geqslant 0\right\}$ and express them with stochastic integrals $\int_{0}^{\cdot} \tilde{Z}_{t}^{x} \sqrt{2 \epsilon\left(X_{t}^{x}\right)} \mathrm{d} B_{t}$.

- The uniform ellipticity of $\epsilon$ allows one to change $\tilde{Z}_{t}^{x} \sqrt{2 \epsilon\left(X_{t}^{x}\right)}$ in $Z_{t}^{x}$.

The BSDE (8) provides a probabilistic interpretation to elliptic semilinear PDEs driven by the differential operator $\mathcal{L}$ :

$$
-\mathcal{L} u(x)+f(x, u(x),(\nabla u \sqrt{2 \epsilon})(x))=0, \quad x \in \mathbb{R}^{d} .
$$

Theorem 3.5. Let $u$ belongs to $\mathcal{C}^{2}\left(\mathbb{R}^{d} \backslash \Gamma\right)$ a solution of Eq.(9), such that for all $x$ in $\mathbb{R}^{d}$

$$
\mathbb{E}\left[\int_{0}^{\infty} e^{\int_{0}^{t} \lambda\left(X_{s}^{x}\right) \mathrm{d} s}\left\|(\nabla u \sqrt{2 \epsilon})\left(X_{s}^{x}\right)\right\|^{2} d t\right]<\infty
$$

Suppose there exists a function $r$ in $\mathcal{C}^{2}$, such that $\forall x \in \mathcal{N}$,

$$
r(x)=\left(\frac{\epsilon_{i n t}}{\epsilon_{e x t}}-1\right) \nabla^{i n t} u(\pi(x)) \cdot n(\pi(x)),
$$

and such that $\hat{u}$ belongs to $\mathcal{C}^{2}\left(\mathbb{R}^{d} \backslash \Gamma\right) \cap W_{\text {loc }}^{2, \infty}\left(\mathbb{R}^{d}\right)$, where

$$
\hat{u}(x):=u(x)-r(x)[\rho(x)]_{+} .
$$

Then, for all $x$ in $\mathbb{R}^{d}$, the couple $\left\{u\left(X_{t}^{x}\right),(\nabla u \sqrt{2 \epsilon})\left(X_{t}^{x}\right)\right\}$ is the solution of BSDE (8); in particular $u(x)=Y_{0}^{x}$.

The proof consists in using a generalised Itô formula proved by Theorem 2.8 in [BCMT10].

Remark 3.6. We should emphasize that $Y_{0}^{x}$ is deterministic as $\left(Y_{t}^{x}\right)_{t}$ is $\mathcal{F}_{t}$-adapted. 


\subsection{Remark}

The regularised $\mathrm{PB}$ equation (Eq. (5)), for $d=3$, is associated to the BSDE $(\infty, 0, f)$ of generator given by

$$
f(t, y)=\tilde{g}\left(X_{t}^{x}\right)-\kappa^{2}\left(X_{t}^{x}\right) \sinh y, \quad x \in \mathbb{R}^{3}, y \in \mathbb{R}, t \in[0, \infty),
$$

where $X_{t}^{x}$ is the solution of the SDE given by Eq. (6), and :

$$
Y_{t}^{x}=Y_{T}^{x}+\int_{t}^{T}\left(\tilde{g}\left(X_{s}^{x}\right)-\kappa^{2}\left(X_{s}^{x}\right) \sinh Y_{s}^{x}\right) \mathrm{d} s-\int_{t}^{T} Z_{s}^{x} \mathrm{~d} B_{s},
$$

for all $t, T$ as $0 \leqslant t \leqslant T$.

Le us remind a result in $\left[\mathrm{BDH}^{+} 03\right]$ :

Theorem 3.7 (Briand and al.). Under Assumptions (h1-h2) with $K, \mu$ and $\lambda$ constants, there exists a unique solution to the BSDE $(\tau, \xi, f)$.

This result does not directly apply to the regularised PB equation (Eq. (5)), on the one hand because of the discontinuities of $\epsilon$ and on the other hand because assuming (h1d) and (h1e) with $\mu$ constant would impose $\mu \geqslant 0$ and $\lambda>0$. It makes difficult (and wrong in general) the verification of the integrability hypothesis that $\mathbb{E}\left[\int_{0}^{\infty} e^{\lambda t}\left|\tilde{g}\left(X_{t}^{x}\right)\right|^{2} \mathrm{~d} t\right]$ is finite.

Remark 3.8. We should emphasize that a result for monotone BSDEs (with constant $\mu=0$ ) is obtained in [Roy04], under the strong hypothesis that $f(t, 0,0) \equiv 0$ a.s., which is not satisfied in our case.

\section{Application to the nonlinear Poisson-Boltzmann equation}

\subsection{Main result}

It is then possible to give the following probabilistic interpretation to the nonlinear Poisson-Boltzmann equation.

Theorem 4.1. The nonlinear Poisson-Boltzmann equation (Eq. (2)) admits the probabilistic interpretation

$$
u(x)=\chi(x) G(x)+Y_{0}^{x}, \quad \forall x \in \mathbb{R}^{3},
$$

where $Y_{0}^{x}$ denotes the solution $\left(Y^{x}, Z^{x}\right)$ of the BSDE (11) corresponding to the regularised Poisson-Boltzmann equation (Eq. (5)).

To prove this result, we need to verify that the unique solution to the regularised Poisson-Boltzmann equation (Eq. (5)) satisfies the assumptions of Theorem 3.5.

\subsection{Existence and uniqueness of the solution}

In [Per12] we show the following existence and uniqueness theorem of a weak solution in the subset $M$ of $H^{1}\left(\mathbb{R}^{3}\right)$,

$$
M:=\left\{v \in H^{1}\left(\mathbb{R}^{3}\right) \mid \sinh v \in L^{2}\left(\mathbb{R}^{3}\right)\right\} .
$$

Theorem 4.2. The regularised Poisson-Boltzmann equation (Eq. (5)) has a unique weak solution $v \in M$. This solution belongs to $\mathcal{C}_{b}^{0}\left(\mathbb{R}^{3}\right) \cap \mathcal{C}^{2}\left(\mathbb{R}^{3} \backslash \Gamma\right)$, and its restriction $v_{\mid \Gamma}$ belongs to $\mathcal{C}^{3}(\Gamma)$. There also exists a function $r$ in $\mathcal{C}^{2}\left(\mathbb{R}^{3}\right)$ such that $\forall x \in \mathcal{N}$

$$
r(x)=\left(\frac{\epsilon_{\mathrm{int}}}{\epsilon_{\mathrm{ext}}}-1\right) \nabla^{\mathrm{int}} v(\pi(x)) \cdot n(\pi(x)),
$$

and such that

$$
\hat{v}(x):=v(x)-r(x)[\rho(x)]_{+}
$$

is in $\mathcal{C}^{2}\left(\mathbb{R}^{3} \backslash \Gamma\right) \cap W_{\text {loc }}^{2, \infty}$. Furthermore, the gradient $\nabla v$ of $v$ belongs to $L^{\infty}$. 
Sketch of proof. (1) The existence and uniqueness of the solution in $H^{1}\left(\mathbb{R}^{3}\right)$ are obtained by an energy minimisation.

(2) The properties $v$ in $\mathcal{C}_{b}^{0}\left(\mathbb{R}^{3}\right) \cap \mathcal{C}^{2}\left(\mathbb{R}^{3} \backslash \Gamma\right)$ and $\hat{v}$ in $W_{\text {loc }}^{2, \infty}$ are proved using similar arguments as in [BCMT10], Appendix A.

(3) The bounded condition of $\nabla v$ is shown in two steps. First, the previous step implies that $\nabla v \in L_{\text {loc }}^{\infty}\left(\mathbb{R}^{3}\right)$. Second, we notice that for $k$ in $\{1, \cdots, 3\}, v$ satisfies:

$$
\epsilon_{\mathrm{ext}} \int_{\Omega_{\mathrm{ext}}} \nabla \partial_{k} v \cdot \nabla \phi+\bar{\kappa} \int_{\Omega_{\mathrm{ext}}} \partial_{k} v \cosh v \phi=0 \quad \forall \phi \in H_{0}^{1}\left(\Omega_{\mathrm{ext}}\right) .
$$

By using the fact that $\bar{\kappa} \cosh \geqslant \bar{\kappa}>0$, we can prove in a very similar way as for the proof of Theorem IX.27 by H. Brezis in [Bre83] that $\nabla u \in L^{\infty}\left(\Omega_{\text {ext }}\right)$.

\subsection{Proof of Theorem 4.1}

The proof follows the following steps.

(1) The following lemma (proved in [Per12]) allows us to verify the integrability Hypothesis (H5) for the generator $f(x, y)=-\kappa^{2}(x) \sinh (y)+\tilde{g}(x)$ of the nonlinear PB equation.

Lemma 4.3. Let $X^{x}$ be a solution of the $S D E(6)$ associated to the operator $\mathcal{L} \cdot:=\nabla(\epsilon \nabla \cdot)$. There exists a choice of $p$ and $q$ positive, such that

$$
\mathbb{E}\left[\int_{0}^{\infty} \exp \left\{\int_{0}^{t} \lambda\left(X_{s}^{x}\right) \mathrm{d} s\right\} \mathrm{d} t\right]<\infty
$$

where $\lambda\left(X_{s}^{x}\right)=p \mathbf{1}_{X_{s}^{x} \in \Omega_{\mathrm{int}}}-q \mathbf{1}_{X_{s}^{x} \in \Omega_{\mathrm{ext}}}$.

So the generator $f$ satisfies Assumptions $(\boldsymbol{H 1}-\boldsymbol{H} \boldsymbol{6})$.

(2) In Section 4.2, we have stated an existence and uniqueness result (Theorem 4.2) for solutions $v$ in $H^{1}$ to the regularised Poisson-Boltzmann equation.

(3) Furthermore, the solution $v$ satisfies the assumptions of Theorem 3.5 which makes the link between PDEs solutions and BSDEs solutions. In particular, Lemma 4.3 and the property $\nabla v \in L^{\infty}$ allow us to verify Assumption (10).

\section{CONCLUSION}

With this probabilistic interpretation, it should be possible to implement new numerical methods for the Poisson-Boltzmann equation. To this aim, an efficient way to simulate the forward process given by Eq. (6) has to be implemented. With more regularities hypothesis, simulation of a Forward-Backward SDE system can be done by quantification ( $c f$. [DM06]) or by a combination of Picard's iterations and an adaptive control variable (cf. [GL10]). However, the adaptation to the PB equation has to be done.

\section{REFERENCES}

[BBC06] Nathan A. Baker, Donald Bashford, and David A. Case. Implicit solvent electrostatics in biomolecular simulation. In New algorithms for macromolecular simulation, volume 49 of Lect. Notes Comput. Sci. Eng., pages 263-295. Springer, Berlin, 2006.

[BCMT10] Mireille Bossy, Nicolas Champagnat, Sylvain Maire, and Denis Talay. Probabilistic interpretation and random walk on spheres algorithms for the Poisson-Boltzmann equation in molecular dynamics. M2AN Math. Model. Numer. Anal., 44(5):997-1048, 2010.

$\left[\mathrm{BDH}^{+}\right.$03] Philippe Briand, Bernard Delyon, Ying Hu, Etienne Pardoux, and Lucrétiu Stoica. $L^{p}$ solutions of backward stochastic differential equations. Stochastic Process. Appl., 108(1):109-129, 2003. 
[Bre83] Haïm Brezis. Analyse fonctionnelle. Collection Mathématiques Appliquées pour la Maîtrise. [Collection of Applied Mathematics for the Master's Degree]. Masson, Paris, 1983. Théorie et applications. [Theory and applications].

[DM06] François Delarue and Stéphane Menozzi. A forward-backward stochastic algorithm for quasi-linear PDEs. Ann. Appl. Probab., 16(1):140-184, 2006.

[GL10] Emmanuel Gobet and Céline Labart. Solving BSDE with adaptive control variate. SIAM J. Numer. Anal., 48(1):257$277,2010$.

[JS03] Jean Jacod and Albert N. Shiryaev. Limit theorems for stochastic processes, volume 288 of Grundlehren der Mathematischen Wissenschaften [Fundamental Principles of Mathematical Sciences]. Springer-Verlag, Berlin, second edition, 2003.

[Par99] Étienne Pardoux. BSDEs, weak convergence and homogenization of semilinear PDEs. In Nonlinear analysis, differential equations and control (Montreal, QC, 1998), volume 528 of NATO Sci. Ser. C Math. Phys. Sci., pages 503-549. Kluwer Acad. Publ., Dordrecht, 1999.

[Per12] Nicolas Perrin. Stochastic methods in molecular dynamics. PhD thesis, Université de Nice Sophia-Antipolis, September 2012.

[Roy04] Manuela Royer. BSDEs with a random terminal time driven by a monotone generator and their links with PDEs. Stoch. Stoch. Rep., 76(4):281-307, 2004. 